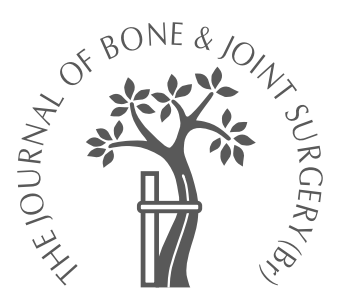

S. W. Suh,

V. U. Shingade,

S. H. Lee,

J. H. Bae,

C. E. Park,

J. Y. Song

From the University of Korea, Seoul, Korea

S. W. Suh, MD, Professor V. U. Shingade, MS, Research Fellow S. H. Lee, MD, Professor J. H. Bae, MD, Senior Registrar

C. E. Park, MD, Senior Registrar

J. Y. Song, MD, Senior Registrar

Department of Orthopaedic Surgery, College of Medicine, Guro Hospital, Korea University, 80 GuroGu, Guro-dong, Seoul, Korea.

Correspondence should be sent to Dr V. U. Shingade at 135 Banerjee Layout, Post Parvatinagar, Nagpur, Maharashtra 440027, India; e-mail: virajshingade@ rediffmail.com

(C)2005 British Editorial Society of Bone and Joint Surgery doi:10.1302/0301-620X.87B4. $15529 \$ 2.00$

$J$ Bone Joint Surg [Br] 2005;87-B:518-22. Received 2 April 2004; Accepted after revision 8 October 2004

\title{
Origin of lumbar spinal roots and their relationship to intervertebral discs
}

\author{
A CADAVER AND RADIOLOGICAL STUDY
}

Previous studies on the anatomy of the lumbar spine have not clarified the precise relationship of the origin of the lumbar roots to their corresponding discs or their angulation to the dural sac. We studied 33 cadavers (25 formalin-preserved and eight freshfrozen) and their radiographs to determine these details.

All cadavers showed a gradual decrease in the angle of the nerve root from L1 to S1. The origin of the root was found to be below the corresponding disc for the L1 to L4 roots. In the formalin-preserved cadavers $8 \%$ of the L5 roots originated above, $64 \%$ below and $28 \%$ at the L4/L5 disc. In the fresh cadavers the values were $12.5 \%, 62.5 \%$ and $25 \%$, respectively. For the S1 root $76 \%$ originated above and $24 \%$ at the L5-S1 disc in the formalin-preserved cadavers and $75 \%$ and $25 \%$, respectively, in the fresh cadavers.

A herniated disc usually compresses the root before division of the root sleeve. Thus, compression of the thecal sac before the origin of the root sleeve is common for L1 to L5 whereas compression at the root sleeve is common for S1.

Our findings are of value in understanding the pathophysiology of prolapse of the disc and in preventing complications during surgery.

As technology has advanced various minimally invasive methods have become popular in spinal surgery. For example, endoscopy is used frequently, particularly in the lumbosacral spine, for the removal of disc protrusions. ${ }^{1}$ With these techniques, however, a precise knowledge of the anatomy of the nerve roots and their relation to the intervertebral disc are essential in order to avoid complications. ${ }^{1,2}$ Published studies on the anatomy of the lumbosacral roots have related their origin to the pedicles and described their oblique course onwards, ${ }^{3-9}$ but the majority of them have not measured the angulation of the nerve roots to the thecal sac at their origin. This is necessary in order to judge their mobility during intra-operative retraction. Earlier research has also failed to identify the precise relationship between the origin of the nerve roots and the corresponding intervertebral disc, which is essential for the understanding of the biomechanics and pathology of prolapse of the disc.

We have, therefore, attempted to define the exact angulation of each lumbar root sleeve to the thecal sac and the relationship of its origin to the corresponding intervertebral disc.

\section{Materials and Methods}

We studied 33 human cadavers, 25 formalinpreserved and eight fresh-frozen. There were
20 women and 13 men with a mean age of 53 years $(32.5$ to 70$)$. Specimens with spinal deformity or previous spinal surgery and those of an older age with loss of disc height or vertebral collapse confirmed from lateral radiographs, were excluded.

In the prone position, we made a posterior midline incision from $\mathrm{T} 12$ to S2. From each end two transverse incisions were made and skin flaps raised on both sides. Muscles and ligaments were detached, spinous processes removed and a wide laminectomy and foraminectomy from T12 to S1 carried out, thereby exposing the dural sac and nerve roots.

The point of origin of each sleeve was noted and marked with ink. Although the root originated at a point, there was always a proximal bulge or prominence. The starting point of this bulge was also marked with ink and the distance between the origin of this bulge and the point of origin of the root sleeve was measured. A long Kirschner wire was then placed over the dura in the midline and small pieces of lead wire positioned along the root sleeves from their points of origin (Fig. 1).

Standard anteroposterior (AP) and lateral radiographs were taken from L1 to S1 (Fig. 2) for every specimen, at a distance of $1 \mathrm{~m} \mathrm{(10 \%}$ magnification) with the lead wires and Kir- 


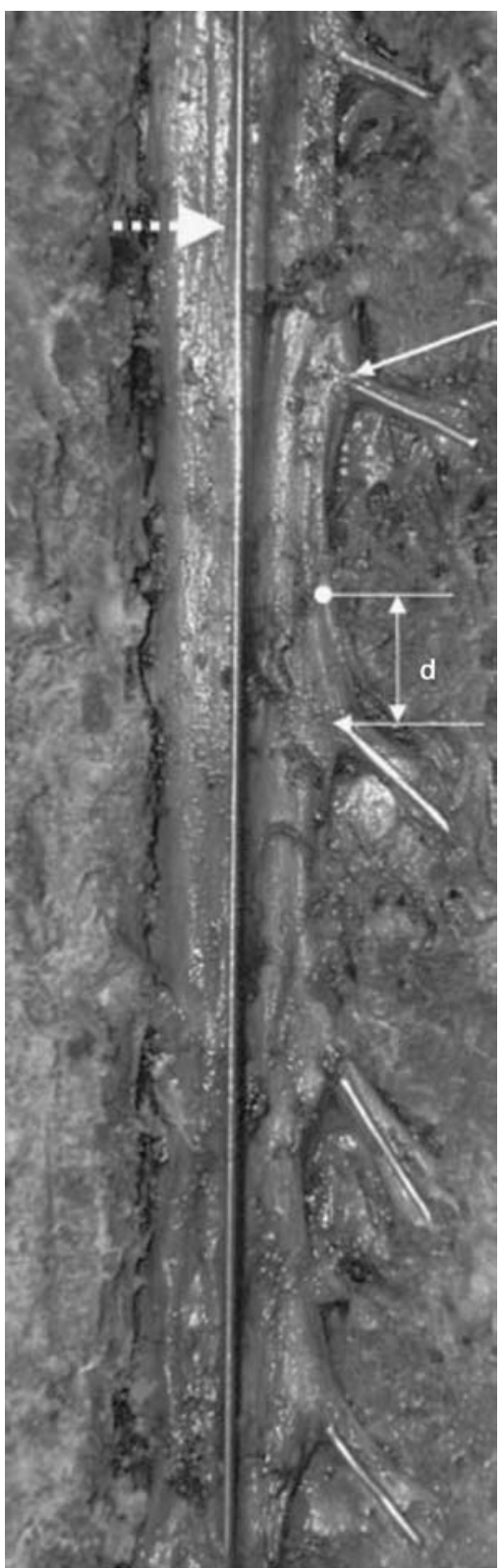

Fig. 1

Photograph of a cadaver, showing the dural sac and nerve roots. Small lead wires are placed along the nerve roots, from the point of origin of the root sleeve (solid long arrow). The distance (d) between the origin of the bulge on the dural sac (round arrowhead), and the origin of the root sleeve was measured. A Kirschner wire, $1 \mathrm{~mm}$ in diameter was placed over the dura in the midline (dotted arrow).

schner wire (K-wire) in situ. The K-wire was removed while taking lateral radiographs to avoid causing overlapping of the lead wires so that the root origins' relationship to the disc could be seen. In order to obtain accurate measurements, the radiographs were scanned, and using the software program Rapidia Version 2.7 (INFINITT, Seoul, Korea), the following parameters were recorded (Fig. 3).

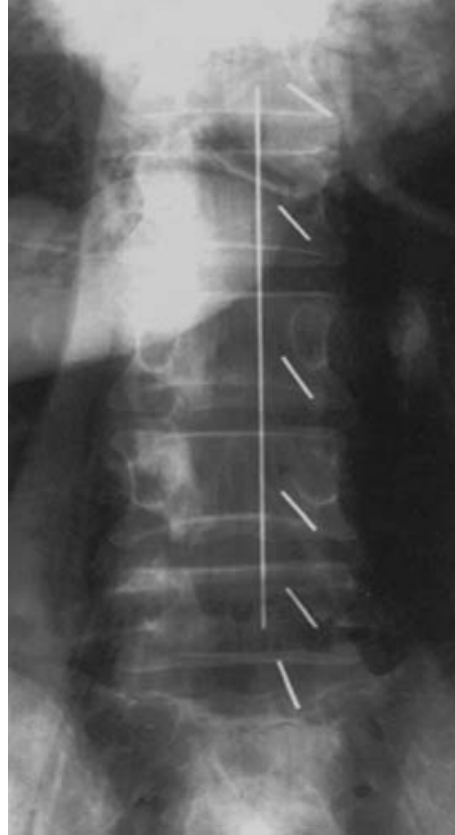

Fig. 2a

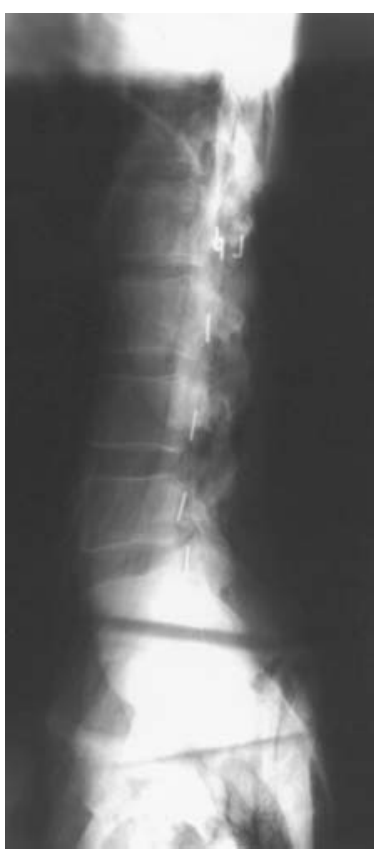

Fig. $2 b$
Radiographs with lead wires and a K-wire in situ, a) AP and b) with lead wires lateral views. The lead wires represent the $\mathrm{L} 1$ to $\mathrm{S} 1$ roots. The medial or superior end of a wire is the origin of the root sleeve.

\section{AP view}

1) The level of the origin of the root sleeve in relation to the corresponding disc (e.g., the L2 root corresponds to the L1/ $\mathrm{L} 2$ disc and the $\mathrm{L} 3$ root to the $\mathrm{L} 2 / 3$ disc).

This was subclassified as (a) above the disc, (b) at the disc and (c) below the disc.

2) The distance (Dc) from the origin of the root sleeve to its corresponding disc. This was measured between two horizontal lines at the vertebral end-plate and at the origin of the root, respectively.

3) The nerve-root angle (A) between the K-wire and each lead wire.

4) The distance $(\mathrm{Db})$ between the origin of the root sleeve and the lower disc. This was measured between two horizontal lines, one at the origin of the root and the other at the vertebral end-plate of the lower disc.

Lateral view (Fig. 4)

1) The disc height (Dh) for each level. This can be measured between the anterior, posterior or central portions of the vertebral bodies. To achieve uniformity we measured it as the distance between the anterior ends of adjacent vertebral end-plates.

2) The vertebral body height (VBh) as measured along the anterior border. A magnification factor of $10 \%$ was taken into account.

For all radiographs, in order to test validity and reproducibility, the measurements on eight fresh cadavers were repeated seven times by different surgeons (SWS, VUS, SHL, JHB, CEP, JYS). The wires were replaced and radiographs taken on each occasion. 


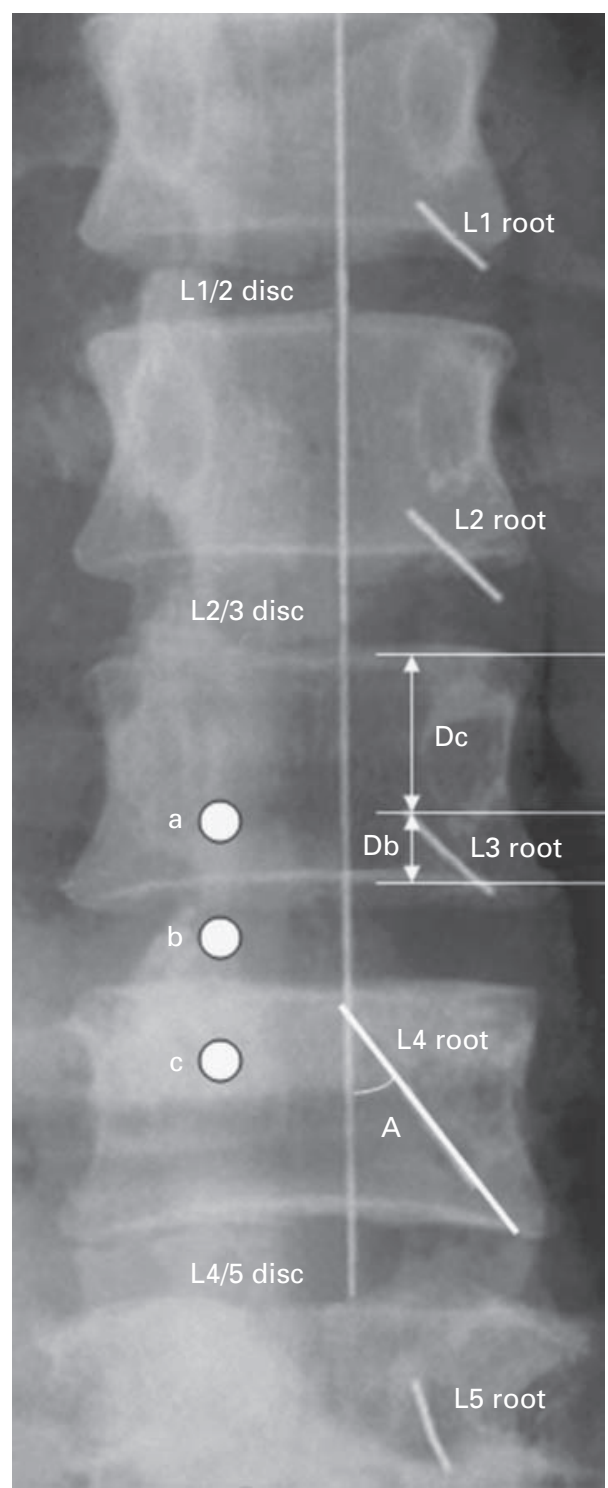

Fig. 3

An AP radiograph showing: 1) the level of origin of the root sleeve in relation to the corresponding disc at (a) above the disc, (b) at the disc, and (c) below the disc. The $L 1$ to $L 5$ roots originate below the corresponding discs); 2) the distance (Dc) from the origin of the root sleeve to its corresponding disc. 3) the nerve-root angle, and 4) the distance (Db) between the origin of the root sleeve and the lower disc $\mathrm{Db}$ for the $\mathrm{L} 3$ root is shown.

Statistical analysis. For detecting differences between observations on formalin-preserved and fresh-frozen cadavers, we used Student's $t$-test. The 95\% confidence interval (CI) of the mean was used with statistical significance set at $\mathrm{p}<0.05$.

\section{Results}

Distance between the origin of the dural bulge and the point of the root sleeve. In both fresh-frozen and formalin-preserved cadavers the mean distance for L1 was $15 \mathrm{~mm}$. Table

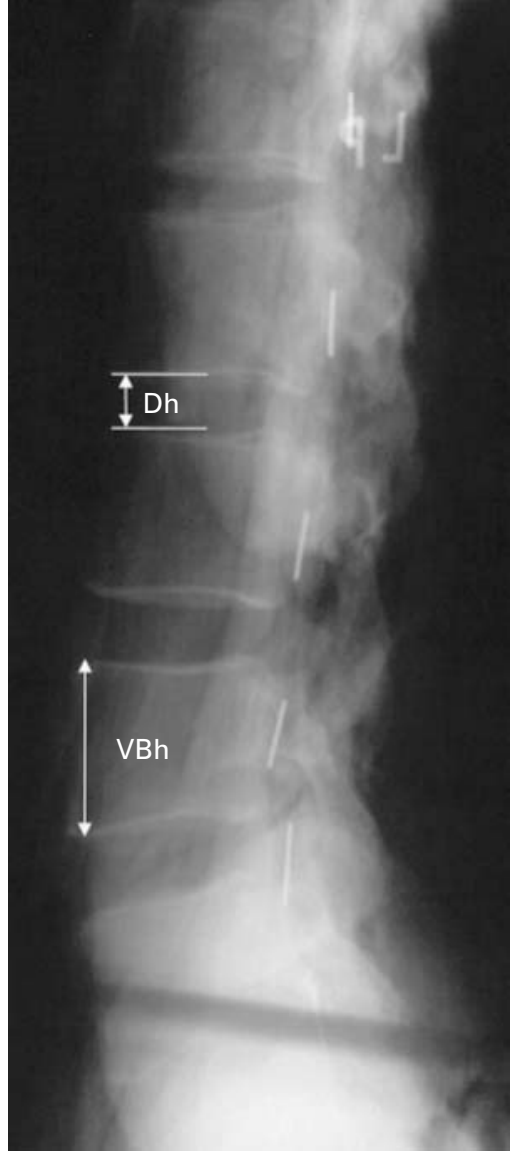

Fig. 4

Lateral radiograph showing the disc height (Dh) measured along the anterior portion of adjacent vertebral end-plates and vertebral body height (VBh) measured along the anterior portion of the body.

I gives the details for L1 to S1. Apart from the L4 root, there was no significant difference between formalin-preserved and fresh-frozen cadavers.

Nerve-root angle. The nerve-root angles for each level (frontal plane) are shown in Table II. For the L1, L2, L4 and L5 roots, there was a statistically significant difference between the mean values of the formalin-preserved and fresh-frozen groups $(\mathrm{p}<0.05)$ and the nerve-root angle gradually decreased from L1 to S1. For L3 and S1 there was no significant difference ( $\mathrm{p}>0.05$ ).

Level of origin of the root sleeve. The L1, L2, L3 and L4 roots in all 33 cadavers had their origin below the corresponding disc level ('c', in Fig. 3). For the L5 and S1 roots, the findings were variable (Table III). For the L1, L2 and L3 roots there was no difference between the mean values of $\mathrm{Dc}$ and $\mathrm{Db}$ for the formalin-preserved and fresh-frozen cadavers (Table IV). There was a significant difference for the $\mathrm{L} 4$ root.

Disc heights at various levels. The mean values at various levels for the different age groups are shown in Table V. 
Table I. Mean $(\mathrm{mm})$ distance between the origin of the dural bulge and the point of origin of the root sleeve for both groups

\begin{tabular}{llll}
\hline & Distance $(\mathbf{m m})$ & & \\
\cline { 2 - 4 } Nerve root & Formalin-preserved & Fresh-frozen & p value $^{*}$ \\
\hline L1 & 15 & 15 & NS \\
L2 & 15 & 16 & NS \\
L3 & 14 & 15 & NS \\
L4 & 13 & 16 & $<0.05$ \\
L5 & 12 & 13 & NS \\
S2 & 12 & 11 & NS \\
\hline
\end{tabular}

* Student's $t$-test; NS, not significant

Table II. Mean $\left({ }^{\circ}\right)$ nerve-root angles for both groups

\begin{tabular}{llll}
\hline & Nerve-root angle $\left({ }^{\circ}\right)$ & & \\
\cline { 2 - 4 } Nerve root & Formalin-preserved & Fresh-frozen & p value \\
\hline L1 & 43 & 45 & $<0.05$ \\
L2 & 37 & 39 & $<0.05$ \\
L3 & 31 & 32 & NS \\
L4 & 29 & 27 & $<0.05$ \\
L5 & 27 & 23 & $<0.05$ \\
S1 & 19 & 18 & NS \\
\hline
\end{tabular}

* Student's $t$-test; NS, not significant

Vertebral heights from L1 to L5. The mean heights for L1, L2, L3, L4 and L5 were $29 \mathrm{~mm}, 30 \mathrm{~mm}, 31 \mathrm{~mm}, 31 \mathrm{~mm}$ and $30 \mathrm{~mm}$, respectively, in all 33 cadavers.

\section{Discussion}

In minimally invasive spinal surgery, direct access to the intervertebral disc is made under radiological control. ${ }^{1,2}$ It is essential to know the precise relationship of the intervertebral disc to the corresponding nerve root.

In the lumbar spine, the root is numbered according to the pedicle beneath which it passes. ${ }^{6}$ Various studies $3,4,10,11$ have mentioned the relationship of the nerve root to the pedicle in detail, but have not revealed the relationship of the origin of the nerve root to the corresponding disc.

When studying a transverse MR scan at the levels of the L4/5 and L5-S1 discs, the root sleeve is not always visible, suggesting a variable origin of the root in the lumbosacral spine. Most of the available studies $^{3-9}$ have not clarified these anatomical details and only a few authors ${ }^{10,11}$ have studied the exact angulation of the nerve roots in the lumbosacral spine. Cohen et $\mathrm{al}^{10}$ in their study of 20 cadav-

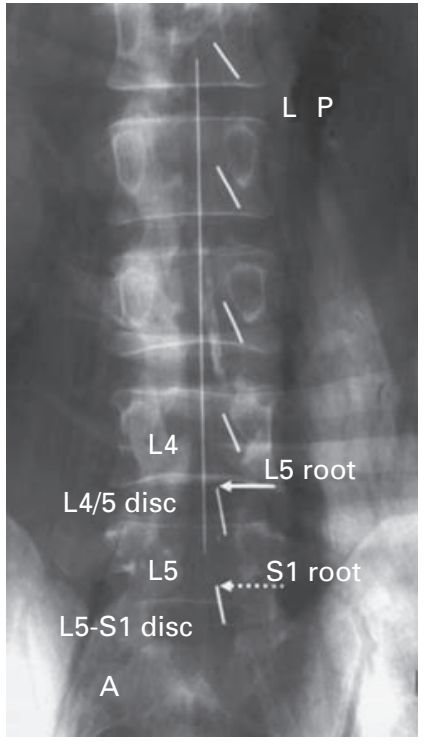

Fig. 5a

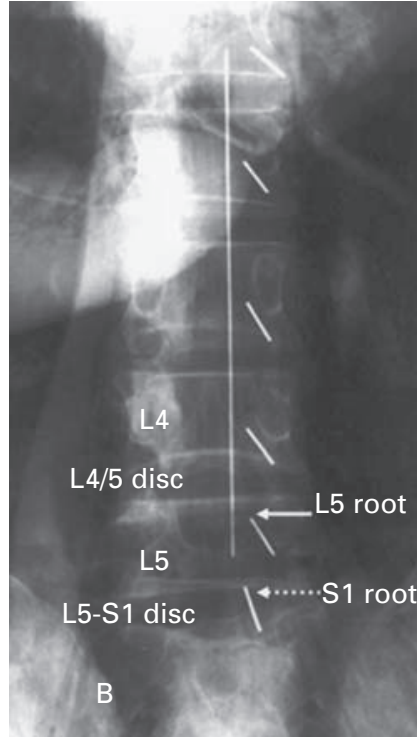

Fig. $5 b$
Radiographs showing that the S1 root generally arises above the L5-S1 disc while the $L 5$ root generally originates below the $L 4 / 5$ disc but this is variable, a) origin of the $L 5$ root at the $L 4 / 5$ disc level and that of $S 1$ above the L5-S1 disc and b) origin of the L5 root below the L4/5 disc and that of $\mathrm{S} 1$ at the level of the L5-S1 disc.

ers stated that $40^{\circ}$ (37 to 41 ) was the mean nerve-root angle for L1 to L5. They also mentioned an acute decrease in this angle for the S1 root. Ebraheim et al, ${ }^{11}$ in their study of 15 cadavers found the variation in the nerve-root angle to be from $33.7^{\circ}$ to $39.2^{\circ}$ from L1 to L5, with the smallest angle at L2 and the largest at L5. Our findings differed from these studies. Although there was a significant difference between the values in the formalin-preserved and fresh cadavers for the L1, L2, L4 and L5 roots, the pattern was the same in both groups. The nerve-root angle gradually decreased from L1 to S1 (Table II, Fig. 1). It is difficult to explain this difference, but we think that geographical variation may be a possible cause since our cadavers had a similar age group to other studies.

We took maximum precautions to identify the precise anatomical relationship of the nerve roots to the discs. Cadavers above 70 years of age were excluded because of anatomical changes, as were those cadavers with a clear-cut loss of disc or body height on lateral radiographs.

Table III. Level of origin of the root sleeve for both groups, by number and percentage

\begin{tabular}{|c|c|c|c|c|c|c|}
\hline \multirow[b]{2}{*}{ Nerve roots } & \multicolumn{2}{|l|}{ Origin above the disc } & \multicolumn{2}{|l|}{ Origin at the disc } & \multicolumn{2}{|l|}{ Origin below the disc } \\
\hline & Formalin-preserved & Fresh-frozen & Formalin-preserved & Fresh-frozen & Formalin-preserved & Fresh-frozen \\
\hline L1 & 00 & 00 & 00 & 00 & 25100 & 8100 \\
\hline L2 & 00 & 00 & 00 & 00 & 25100 & 8100 \\
\hline L3 & 00 & 00 & 00 & 00 & 25100 & 8100 \\
\hline L4 & 00 & 00 & 00 & 00 & 25100 & 8100 \\
\hline L5 & 28 & 112.5 & 728 & 225 & $16 \quad 64$ & $5 \quad 62.5$ \\
\hline $\mathrm{S} 1$ & 1976 & 675 & 624 & 225 & 0 & 0 \\
\hline
\end{tabular}


Table IV. Mean $(\mathrm{mm})$ values of the distance between the origin of the root sleeve from the corresponding disc (Dc) and from the disc below (Db)

\begin{tabular}{llllll}
\hline & \multicolumn{2}{l}{ Distance $(\mathbf{m m})$ from corresponding disc $(\mathbf{D c})$} & & \multicolumn{2}{l}{ Distance $(\mathbf{m m})$ from disc below $(\mathrm{Db})$} \\
\cline { 2 - 3 } \cline { 5 - 6 } Origin of the root sleeve for & Formalin-preserved & Fresh-frozen & & Formalin-preserved & Fresh-frozen \\
\hline L1 & 16 & 16 & 12 & 11 \\
L2 & 15 & 14 & 14 & 14 \\
L3 & 13 & 14 & 18 & 17 \\
L4 & 7 & 9 & 24 & 22 \\
\hline
\end{tabular}

Table V. The mean $(\mathrm{mm})$ disc heights $(\mathrm{Dh})$ from T12-L1 to L5-S1

\begin{tabular}{lccccc}
\hline & \multicolumn{3}{l}{ Age group (yrs) } & & \\
\cline { 2 - 6 } & $\mathbf{3 2}$ to $\mathbf{4 0}$ & $\mathbf{4 1}$ to $\mathbf{5 0}$ & $\mathbf{5 1}$ to $\mathbf{6 0}$ & $\mathbf{6 1}$ to $\mathbf{7 0}$ & Mean height (mm) \\
\hline Number of cadavers & 3 & 3 & 16 & 11 & 8 \\
T12 to L1 & 9 & 7 & 8 & 8 & 11 \\
L1 to L2 & 11 & 12 & 11 & 10 & 11 \\
L2 to L3 & 12 & 11 & 10 & 11 & 13 \\
L3 to L4 & 12 & 14 & 14 & 12 & 14 \\
L4 to L5 & 15 & 14 & 15 & 12 & 16 \\
L5 - S1 & 16 & 16 & 17 & 15 & \\
\hline
\end{tabular}

Because of the lumbar lordosis, disc height varies according to the part of the vertebral body from which the measurements were taken. In order to achieve uniformity all disc heights were measured along the anterior portion of the body.

Our results (Table III) indicated that for L1 to L4 levels, the origin of the root is always below the level of the corresponding disc (Fig 5). Although the L5 root commonly arises below the L4/5 disc, it may also arise either at or above the disc. This explains the intermittent visibility of the L5 root sleeves on transverse sections of MR scans at the L $4 / 5$ disc level. The S1 root usually originates above the L5-S1 disc, but is sometimes at the level of the disc.

McCulloch and Transfeldt ${ }^{6}$ stated that the proximal lumbar roots are more horizontal making L1 less mobile than S1. Our study (Table II), confirmed that the upper roots are more horizontal and less mobile compared with the lower roots and therefore require meticulous retraction.

The relationship of the level of the disc to the origin of the root (Table II), explains why a herniated disc usually compresses the dural sac before comprising the root sleeve. Compression of the thecal sac is common for L1 to L5 roots but compression of the root sleeve is found for the $\mathrm{S} 1$ root and may sometimes occur for the L5 root. Therefore, the shoulder type of disc prolapse is common for L1 to L5, and the axillary type for $\mathrm{S} 1$.

Although the root sleeve originates distal to the disc from L1 to L5, the actual bulge of its origin starts proximally at the level of the disc (Fig. 1, Tables I and IV). The herniated disc compresses the bulge of the lower root, thereby explaining lower root compression symptoms, even though the origin of the root is below the disc. The distance between the origin of the dural bulge and the point of origin of the root sleeve (Fig. 1) explains this phenomenon.

The major weakness of our study is the use of preserved cadavers. Formalin affects the anatomy and we therefore extended our dissections to include fresh-frozen cadavers.

There was a significant difference for the mean values of d (Fig. 1), Dc, and Db (Fig. 3) for the L4 root between the formalin and fresh cadavers. This could be due not only to the formalin but to the small sample size of fresh cadavers.

From the L1 to S1 levels, the pattern of the nerve-root angle, and the relationship of the origin of the root to its corresponding and inferior disc were similar for all cadavers. Our measurements are easily reproducible. Except for manual measurement of the bulged portion of the dura, all other measurements were calculated from scanner radiographs using the computer program and thereby avoiding manual error.

No benefits in any form have been received or will be received by a commercial party related directly or indirectly to the subject of this article.

\section{References}

1. Regan JJ. Minimally invasive techniques in lumbar spine surgery. In: Vaccaro AR, Betz RR, Zeidman SM, eds. Principles and practice of spine surgery. First ed. Philadelphia: Mosby, 2003:265-73.

2. Mathews HH, Long BH. Posterior minimally invasive techniques. In: Vaccaro AR, Betz RR, Zeidman SM, eds. Principles and practice of spine surgery. First ed. Philadelphia: Mosby, 2003:283-94

3. Rausching W. Normal and pathologic anatomy of the lumbar root canals. Spine 1987:12:1008-19.

4. Crock HV. Normal and pathological anatomy of the lumbar spinal nerve root canals. J Bone Joint Surg [Br] 1981;63-B:487-90.

5. Niel N, Feuerstein M. Angulated course of spinal nerve roots. J Neurosurg 1970;32: 349-52.

6. McCulloch JA, Transfeldt EE. Macnab's backache. Third ed. Baltimore: Lippincott, Williams \& Wilkins, 1997:1-74.

7. Parke WW. In: Herkowitz HN, Garfin SR, Balderston RA, et al, eds. Rothman-Simeone: the spine. Vol. 1 Fourth ed. Philadelphia: W. B. Saunders Co, 1999:29-73.

8. Borenstein DG, Wiesel SW, Boden SD. Low back pain: medical diagnosis and comprehensive management. Second ed. Philadelphia: W. B. Saunders Co, 1995:3-21.

9. Hay MC. Anatomy of the lumbar spine. Med J Aust 1976;1:874-6.

10. Cohen MS, Wall EJ, Brown RA, Rydevik B, Garfin SR. Cauda equina anatomy II: extrathecal nerve roots and dorsal root ganglia. Spine 1990;15:1248-51.

11. Ebraheim NA, Xu R, Darwich M, Yeasting RA. Anatomic relations between the lumbar pedicle and the adjacent neural structures. Spine 1997;22:2388-41. 